\title{
Reliability Analysis of 6-Component Star Markov Repairable System with Spatial Dependence
}

\author{
Liying Wang, ${ }^{1}$ Qing Yang, ${ }^{2}$ and Yuran Tian' \\ ${ }^{1}$ Department of Mathematics \& Physics, Shijiazhuang Tiedao Institute, Shijiazhuang 050043, China \\ ${ }^{2}$ School of Software, Central South University, Changsha 410075, China \\ Correspondence should be addressed to Liying Wang; wly_sjz@sohu.com
}

Received 14 November 2016; Accepted 16 January 2017; Published 5 February 2017

Academic Editor: Huanqing Wang

Copyright (c) 2017 Liying Wang et al. This is an open access article distributed under the Creative Commons Attribution License, which permits unrestricted use, distribution, and reproduction in any medium, provided the original work is properly cited.

\begin{abstract}
Star repairable systems with spatial dependence consist of a center component and several peripheral components. The peripheral components are arranged around the center component, and the performance of each component depends on its spatial "neighbors." Vector-Markov process is adapted to describe the performance of the system. The state space and transition rate matrix corresponding to the 6-component star Markov repairable system with spatial dependence are presented via probability analysis method. Several reliability indices, such as the availability, the probabilities of visiting the safety, the degradation, the alert, and the failed state sets, are obtained by Laplace transform method and a numerical example is provided to illustrate the results.
\end{abstract}

\section{Introduction}

In a multicomponent system, the failure of one component can reduce the redistribution of the system loading (Yu et al. [1]). Furthermore, many empirical researches have indicated that the workload strongly affects a component's failure rate and an increased load induces a higher failure rate [2-5]. Thus how to describe the dependency among components in a system has been an interesting topic. Various types of dependence, such as Markov dependence [6-8], redundant dependence, $[9,10]$, common cause failure [11-13], sequencedependent failures (Xing et al. [14]), propagated failures with global or selective effect $[15,16]$, correlated failures [17], economic dependence (Zhou et al. [18]), and historydependence (Wang and Cui [19]), have been considered.

Recently, Wang and Si [20] proposed a spatial dependence circular system based on the operating process of large intelligent air conditioning system. The large intelligent air conditioning system usually consists of several subsystems (components) and these components are arranged in a circle. If a component fails, its two "neighbors" (left and right) will detect the change and take the load. Therefore, the loads in this kind of system are distributed among the failed component's "neighbors" instead of all of the system's surviving components and the performance of each component depends on its spatial "neighbors." And this kind of dependence is different from those in literatures and the authors name it as spatial dependence. Wang et al. [21] extended the system into the case that the performance of each component depends on its four spatial "neighbors." In the reliability engineer, the spatial pattern among components might be a line, a lattice, a central component surrounded by a peripheral of other components, and so on. We consider a star system in this paper. In the system, one component is at the center and the others are peripheral. The star system can also be seen in warehouse storage systems. In these systems, a bigger warehouse is usually surrounded by several smaller warehouses. When the bigger warehouse fails, all the smaller warehouses will take the load. While a smaller warehouse fails, the load will be redistributed among the bigger warehouse and the smaller warehouses near to it. The phenomenon may be found in local area network systems.

The paper is organized as follows. A 6-component star Markov repairable system with spatial dependence is formulated in Section 2 and the states of systems are given in this section. Section 3 concentrates on the state transition 
analysis of the system. Availability of the system is obtained in Section 4. A numerical example is provided in Section 5. Availability, probabilities of visit to the safety, the degradation, the alert, and the failed state sets are obtained. Finally, a conclusion is given.

\section{System Formulation}

2.1. 6-Component Star Markov Repairable System with Spatial Dependence. Assumptions for a star Markov repairable system with spatial dependence are stated below.

(1) A star Markov repairable system with spatial dependence consists of $n$ components that are divided into two types (peripheral and center) and arranged in a star. $n-1$ peripheral components are identical and are arranged in a polygon. One component is in the center of the polygon. Each component has two states, functional and failed. The $n-1$ peripheral components are numbered as $1,2, \ldots, n-1$ and the center component is named as $n$. For $k=2, \ldots, n-$ 2 , the "neighbors" of component $k$ are components $k+1, k-1$, and $n$. The "neighbors" of component 1 are components $2, n-1$, and $n$. Similarly the "neighbors" of component $n-1$ are components 1 , $n-2$, and $n$, while the "neighbors" of component $n$ are components $1,2, \ldots, n-1$.

(2) Each component has two states, functional and failed. They may adjust their operating rates according to the states of their "neighbors" when they are functional. Let $l(0 \leq l \leq 3)$ denote the number of a peripheral component's "neighbors" that are in failed state. Assume that the lifetime duration of a peripheral component is also an exponential random variable with parameter $\lambda_{l}\left(0<\lambda_{0}<\lambda_{1}<\lambda_{2}<\lambda_{3}\right)$. Let $m(0 \leq m \leq n-1)$ denote that the number of peripheral components is in failed state. Assume that the lifetime duration of component $n$ is also an exponential random variable whose failure rate varies with $m$. When $m \leq[(n-1) / 2]$ (the biggest number that is not more than $(n-1) / 2)$, the failure rate is $\lambda_{4}$. If $m>[(n-1) / 2]$, the failure rate is $\lambda_{5}\left(0<\lambda_{4}<\lambda_{5}<\right.$ $\left.\lambda_{1}\right)$.

(3) The system and its components are repairable and the repair is assumed to be perfect. Each component is assumed to have its own dedicated repair crew. Upon failure, repair can be carried out immediately. The repair time of each component is exponentially distributed with repair rate $\mu$ and is independent of the lifetime durations. In the initial instant, every component is new.

The system proposed in this paper is different from those in Wang and Si [20] and Wang et al. [21]. The star system consists of two types (peripheral and center) of components arranged in a star, instead of identical components arranged in a circle. Furthermore, the performance of the component in the center depends on all the components around it.

The performance of a star repairable system with spatial dependence is determined not only by the states of its components but also by the spatial pattern among components. For a large system, the number of states may be overwhelming. We will discuss the reliability of the 6-component star Markov repairable system with spatial dependence in this paper.

Without special statement, we will use "the system" to denote the 6-component star Markov repairable system with spatial dependence defined above.

2.2. States of the System. We use $\mathbf{X}^{(6)}(t)=\left[X_{6}(t): X_{1}(t)\right.$, $\left.X_{2}(t), \ldots, X_{5}(t)\right](t \geq 0)$ to denote the state of the system, where $X_{i}(t)=1$ or $0(i \in\{1,2, \ldots, 6\})$ accordingly as component $i$ is functioning or failed. Since all the random variables involved in the system are exponentially distributed, $\mathbf{X}^{(6)}(t)$ is a continuous-time Markov process, with state space $S=\{0,1\}^{6}$. Obviously, $2^{6}$ elements are included in $S$ and we call them the elementary states. Taking into account the symmetries of the star configuration, $S$ can be reduced to $\widetilde{S}$ with the sixteen states. The sixteen states are shown in Figure 1.

State $0,[1: 1,1,1,1,1]$, represents that all components are working. State $1,[1: 0,1,1,1,1]$, denotes that component $n$ is working and one of the peripheral components fails. It can occur in five ways. State $2,[0: 1,1,1,1,1]$, represents that component $n$ fails and the $n-1$ peripheral components are working. State $3,[0: 0,1,1,1,1]$, denotes that component $n$ fails and one of peripheral components fails. It can occur in five ways. State $4,[1: 0,0,1,1,1]$, represents that component $n$ is working and two adjacent peripheral components fail. It can occur in 4 ways and the others are $[1: 1,1,0,0,1],[1$ : $1,1,1,0,0]$, and $[1: 0,1,1,1,0]$. State $5,[1: 0,1,0,1,1]$, represents that component $n$ is working and two peripheral components which are separated by a functional component fail. It can occur in five ways and the others are $[1: 1,0,1,0$, $1],[1: 1,1,0,1,0],[1: 0,1,1,0,1]$, and $[1: 1,0,1,1,0]$.

States 6 and 7 can be defined by changing component $n$ 's state to be 0 and leaving the other components' states unchanged in States 5 and 6, respectively. Similarly, we can define States $8-15$ based on the above states. Let $\widetilde{\mathbf{X}}^{(6)}(t)$ be the vector-valued continuous-time Markov process with state space $\widetilde{S}$. We call it the aggregated process associated with $\mathbf{X}(t)$ (Ball et al. [22]).

\section{State Transition Analysis of the System}

The evolution of the Markov system $\widetilde{\mathbf{X}}^{(6)}(t)$ is determined by transitions among the sixteen states. Taking into account the states of center and several peripheral components, the state transitions of the system within the time $\Delta t$ can be discussed. They are shown in Figure 2.

Sorting the states in $\widetilde{S}$ as state 0 , state 1 , state 2 , state 3 , state 4 , state 5 , state 6 , state 7 , state 8 , state 9 , state 10 , state 11 , state 


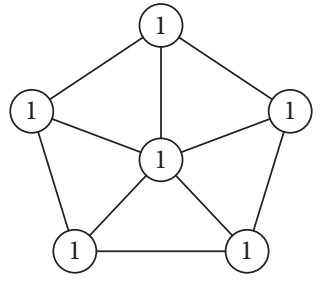

State 0

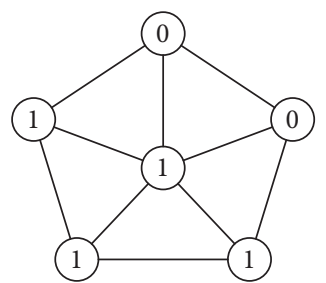

State 4

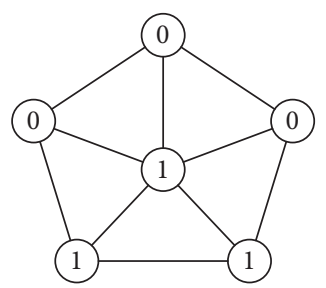

State 8

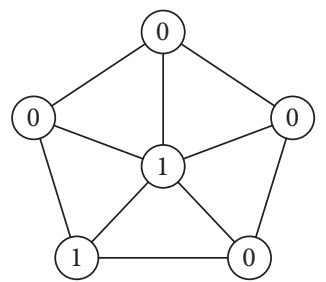

State 12

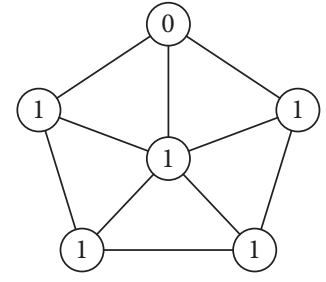

State 1

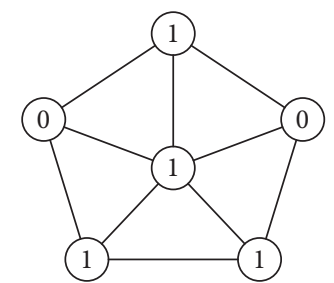

State 5

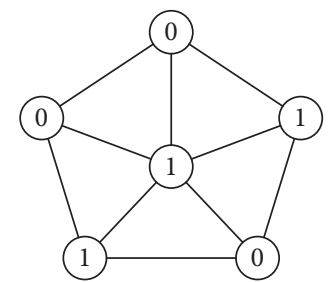

State 9

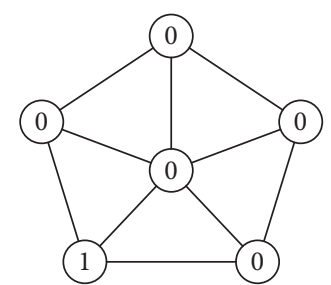

State 13

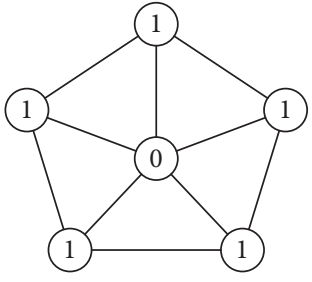

State 2

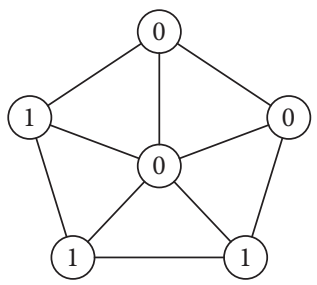

State 6

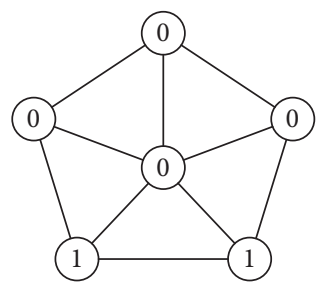

State 10

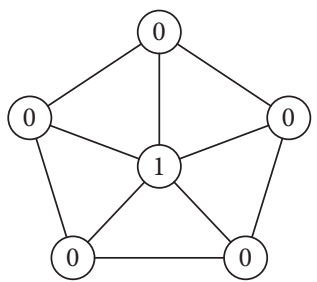

State 14

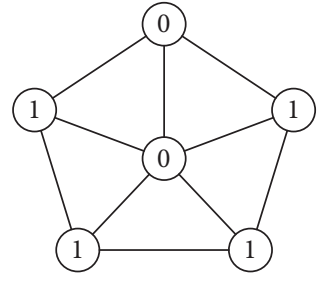

State 3

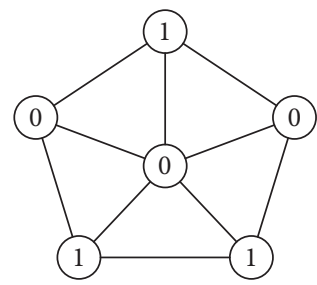

State 7

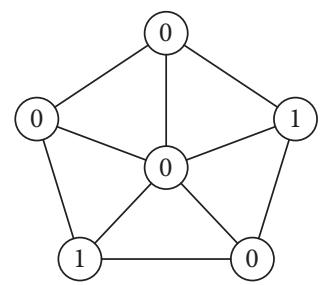

State 11

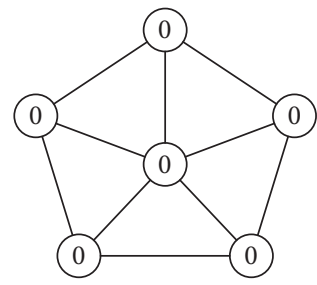

State 15

FIGURE 1: 16 states of the system.

12 , state 13 , state 14 , and state 15 , we can get the transition rate where matrix of $\widetilde{\mathbf{X}}^{(6)}(t), Q$, from Figure 2. Partition $Q$ is as

$$
Q=\left[\begin{array}{ll}
A & B \\
C & D
\end{array}\right],
$$

A

$$
=\left[\begin{array}{cccccccc}
-5 \lambda_{0}-\lambda_{4} & 5 \lambda_{0} & \lambda_{4} & 0 & 0 & 0 & 0 & 0 \\
\mu & -\mu-\lambda_{4}-2 \lambda_{0} & 0 & \lambda_{4} & 2 \lambda_{1} & 2 \lambda_{0} & 0 & 0 \\
\mu & 0 & -\mu-5 \lambda_{1} & 5 \lambda_{1} & 0 & 0 & 0 & 0 \\
0 & \mu & \mu & -2 \mu-2 \lambda_{2}-2 \lambda_{1} & 0 & 0 & 2 \lambda_{2} & 2 \lambda_{1} \\
0 & 2 \mu & 0 & 0 & -2 \mu-\lambda_{4}-2 \lambda_{1}-\lambda_{0} & 0 & 0 \\
0 & 2 \mu & 0 & 0 & 0 & \mu & -2 \mu-\lambda_{4}-2 \lambda_{1}-\lambda_{2} & 0 \\
0 & 0 & 0 & 2 \mu & 0 & \mu & -3 \mu-2 \lambda_{2}-\lambda_{1} & 0 \\
0 & 0 & 0 & 2 \mu & 0 & 0 & -3 \mu-\lambda_{3}-2 \lambda_{2}
\end{array}\right],
$$


$B=\left[\begin{array}{cccccccc}0 & 0 & 0 & 0 & 0 & 0 & 0 & 0 \\ 0 & 0 & 0 & 0 & 0 & 0 & 0 & 0 \\ 0 & 0 & 0 & 0 & 0 & 0 & 0 & 0 \\ 0 & 0 & 0 & 0 & 0 & 0 & 0 & 0 \\ 2 \lambda_{1} & \lambda_{0} & 0 & 0 & 0 & 0 & 0 & 0 \\ \lambda_{2} & 2 \lambda_{1} & 0 & 0 & 0 & 0 & 0 & 0 \\ 0 & 0 & 2 \lambda_{2} & \lambda_{1} & 0 & 0 & 0 & 0 \\ 0 & 0 & \lambda_{3} & 2 \lambda_{2} & 0 & 0 & 0 & 0\end{array}\right]$,

$C=\left[\begin{array}{cccccccc}0 & 0 & 0 & 0 & 2 \mu & \mu & 0 & 0 \\ 0 & 0 & 0 & 0 & \mu & 2 \mu & 0 & 0 \\ 0 & 0 & 0 & 0 & 0 & 0 & 2 \mu & \mu \\ 0 & 0 & 0 & 0 & 0 & 0 & \mu & 2 \mu \\ 0 & 0 & 0 & 0 & 0 & 0 & 0 & 0 \\ 0 & 0 & 0 & 0 & 0 & 0 & 0 & 0 \\ 0 & 0 & 0 & 0 & 0 & 0 & 0 & 0 \\ 0 & 0 & 0 & 0 & 0 & 0 & 0 & 0\end{array}\right]$,

$D=\left[\begin{array}{cccccccc}-3 \mu-\lambda_{5}-2 \lambda_{1} & 0 & \lambda_{5} & 0 & 2 \lambda_{1} & 0 & 0 & 0 \\ 0 & -3 \mu-\lambda_{5}-2 \lambda_{2} & 0 & \lambda_{5} & 2 \lambda_{2} & 0 & 0 & 0 \\ \mu & 0 & -4 \mu & 0 & 0 & 0 & 0 & 0 \\ 0 & \mu & 0 & -4 \mu & 0 & 0 & 0 & 0 \\ 2 \mu & 2 \mu & 0 & 0 & -4 \mu-\lambda_{5}-\lambda_{2} & \lambda_{5} & \lambda_{2} & 0 \\ 0 & 0 & 2 \mu & 2 \mu & \mu & -5 \mu & 0 & 0 \\ 0 & 0 & 0 & 0 & 5 \mu & 0 & -5 \mu-\lambda_{5} & \lambda_{5} \\ 0 & 0 & 0 & 0 & 0 & 5 \mu & \mu & -6 \mu\end{array}\right]$.

\section{Availability of the System}

Assume that $\mathbf{P}(t)=\left(P_{0}(t), P_{1}(t), \ldots, P_{15}(t)\right)$ denotes the state probabilities of the $\widetilde{\mathbf{X}}^{(6)}(t)$. $\mathbf{P}(t)$ can be found from the Kolmogorov equations

$$
\frac{d \mathbf{P}(t)}{d t}=\mathbf{P}(t) Q,
$$

given the initial conditions $\mathbf{P}(0)=(1,0,0,0,0,0,0,0,0,0$, $0,0,0,0,0,0)$.

System (3) can be solved analytically by using Laplace transforms. Using the transform and taking into account the initial conditions, the equations can be represented in the form of linear algebraic equations (Widder [23])

$$
\mathbf{P}^{*}(s)=\mathbf{P}(0)(s \mathbf{I}-Q)^{-1},
$$

where $\mathbf{P}^{*}(s)$ is the Laplace transform of $\mathbf{P}(t)$.

Assume that the system output in the cases that the center component fails and the number of failed peripheral components is not less than three cannot satisfy the demand of customers. So the unacceptable state set of the system is $F=$ \{state 10 , state 11 , state 13 , state 15 . The system entrance into the state in unacceptable set $F$ constitutes a failure (Lisnianski and Levitin [24]). Let $A(t)$ is the probability that the system

is functioning at time $t$, that is, the instantaneous availability of the system; then its Laplace transform can be obtained by

$$
A^{*}(s)=\mathbf{P}(0)(s \mathbf{I}-Q)^{-1} \boldsymbol{\mu},
$$

where $\boldsymbol{\mu}=(1,1,1,1,1,1,1,1,1,1,0,0,1,0,1,0)^{T}$. Using the inverse Laplace transforms, one can get $A(t)$.

Using $\pi$ to represent the stationary distribution of the system, it can be found by solving equations

$$
\begin{aligned}
& \pi Q=(0,0,0,0,0,0,0,0,0,0,0,0,0,0,0,0,0,0,0,0,0,0, \\
& \quad 0,0), \\
& \pi \mathbf{e}=1,
\end{aligned}
$$

where $\mathbf{e}$ is a 16-dimensional row vector and the elements are 1. The asymptotic availability $A=\pi \mu$.

\section{A Numerical Example}

5.1. Instantaneous Availability. Consider the 6-component star Markov repairable systems with spatial dependence introduced in Section 2.1. The system has the following failure rates $\lambda_{0}=1 / 4, \lambda_{1}=2 / 3, \lambda_{2}=3 / 4, \lambda_{3}=1, \lambda_{4}=$ $1 / 8, \lambda_{5}=1 / 5$, and repair rate $\mu=1 / 2$. Substituting them 


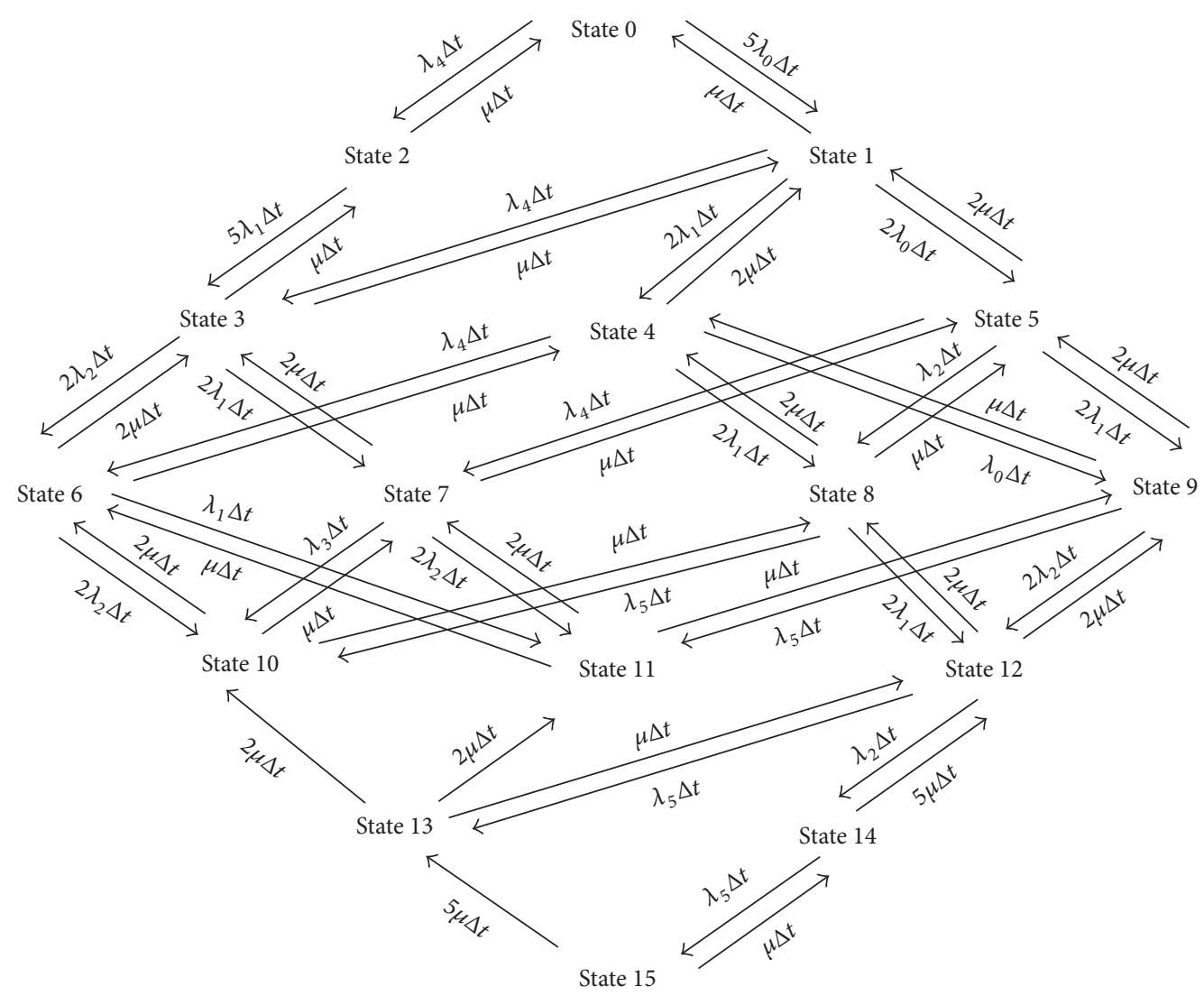

Figure 2: State space diagram of the system.

into expressions in Sections 3 and 4 and taking Laplace and inverse Laplace transforms using Matlab, $A(t)$ can be given. Solving (6), we can get the asymptotic availability $A=0.8591$.

In order to make contrast, we consider the classical 6component parallel (spatial independent) Markov repairable systems. Assume that components of the system are identical and independent. Their life and repair times are, respectively, exponentially distributed with $\lambda_{0}=1 / 4$ and $\mu=1 / 2$. Define the state of the system as the number of failed components. It is easy to get the instantaneous availability $\bar{A}(t)$. The asymptotic availability $\bar{A}=0.9986 . A(t)$ and $\bar{A}(t)$ are presented in Figure 3.

Figure 3 shows that, under the conditions that components have the same lifetime and repair time, the steady-state availability of the system with spatial dependence is less than that of independent system. Furthermore, the dependent system tends to use longer time to get stability.

5.2. Probability of Visit to Four State Sets. According to the number of peripheral failed components and the state of the center component, we also divide the 16 states of the system with spatial dependence into the four categories, security state set $S$, deteriorated state set $D$, and warning (alert) state set $W$, where $S=$ \{state 0 , state 1 , state 2 , state 3 , state 4 , state $5\}, D=\{$ state 8 , state 9 , state 12$\}$, and $W=$ state 6 , state 7 , state 14$\}$.
From (4), one can obtain the Laplace transforms of the instantaneous probabilities of the system in the security, degraded, warning, and failure state sets

$$
\begin{gathered}
S^{*}(s)=\mathbf{P}(0)(s \mathbf{I}-Q)^{-1} \boldsymbol{\mu}_{1}, \\
D^{*}(s)=\mathbf{P}(0)(s \mathbf{I}-Q)^{-1} \boldsymbol{\mu}_{2}, \\
W^{*}(s)=\mathbf{P}(0)(s \mathbf{I}-Q)^{-1} \boldsymbol{\mu}_{3}, \\
F^{*}(s)=\mathbf{P}(0)(s \mathbf{I}-Q)^{-1} \boldsymbol{\mu}_{4},
\end{gathered}
$$

where $\boldsymbol{\mu}_{1}=(1,1,1,1,1,1,0,0,0,0,0,0,0,0,0,0)^{T}, \boldsymbol{\mu}_{2}=(0,0$, $0,0,0,0,0,0,1,1,0,0,1,0,0,0)^{T}, \boldsymbol{\mu}_{3}=(0,0,0,0,0,0,1,1,0$, $0,0,0,0,0,1,0)^{T}$, and $\boldsymbol{\mu}_{4}=(0,0,0,0,0,0,0,0,0,0,1,1,0,1$, $0,1)^{T}$. On inversion, one can get the instantaneous probabilities of visiting the four state sets. The curves are shown in Figure 4. The asymptotic probabilities $S=\pi \mu_{1}=0.3759, D=$ $\pi \mu_{2}=0.3673, W=\pi \mu_{3}=0.1159$, and $F=\pi \mu_{4}=0.1409$.

\section{Conclusion}

Motivated by the operating processes of large intelligent air conditioning systems, warehouse storage systems, and network transmission systems, a stochastic system with interacting components is proposed in this paper. The results 


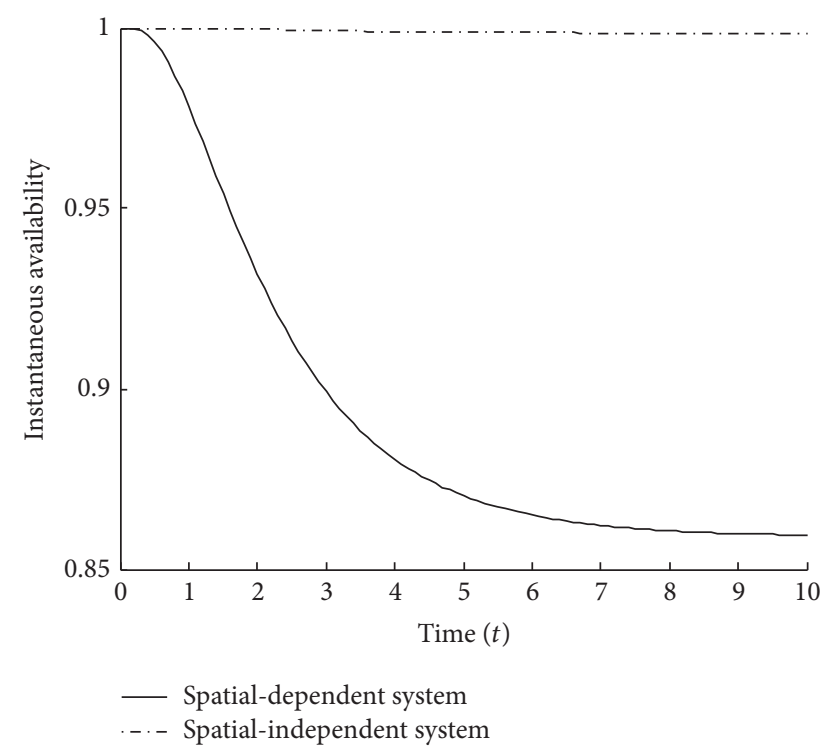

FIGURE 3: Instantaneous availability of the two 6-component systems.

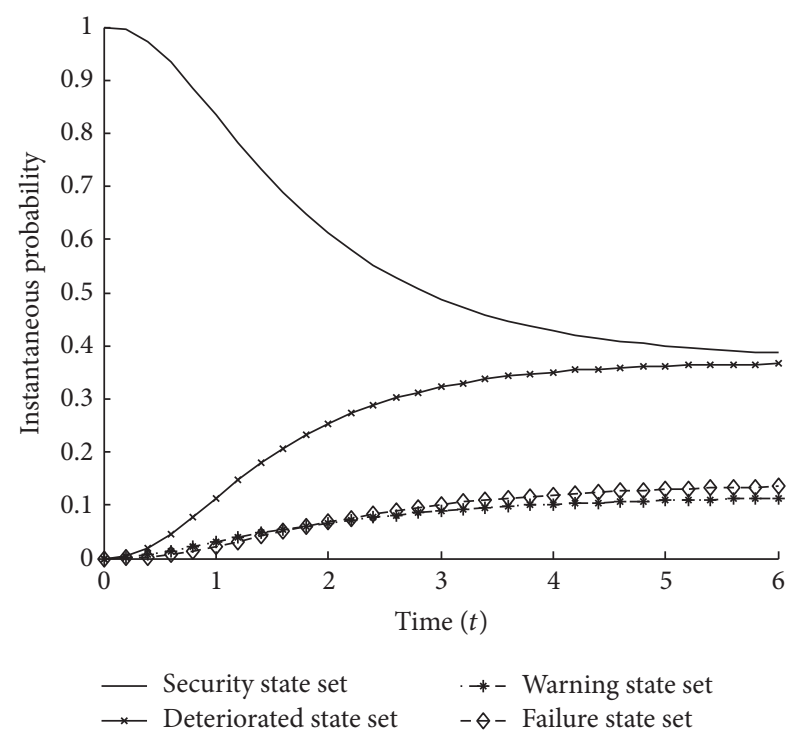

FIGURE 4: Instantaneous visiting probabilities to four state sets.

obtained in this paper may be useful in the reliability evaluation of them. The performance of the system is described by Markov stochastic process. It extends the classical Markov repairable system to one whose $n$ components are arranged in a star. $n-1$ components are identical and are arranged in a polygon. One component is in the center of the polygon. The failure rates of all components vary with the number of failed components in their "neighbors." Availability, probabilities of visiting security, degraded, warning (alert), and failure state sets of the system have also been considered.

We started with a relatively simple model and more complex and realistic models will be built in the future. Lattice systems with spatial dependence, semi-Markov systems with spatial dependence, and systems with other spatial patterns, such as a rosette and three-dimensional pattern, are worth being considered.

\section{Competing Interests}

The authors declare that they have no competing interests.

\section{Acknowledgments}

This work is supported partly by the NSF of China Grants 71201111 and 11471218 and Hebei Province (A2015210103).

\section{References}

[1] H. Yu, C. Chu, E. Châtelet, and F. Yalaoui, "Reliability optimization of a redundant system with failure dependencies," Reliability Engineering and System Safety, vol. 92, no. 12, pp. 1627-1634, 2007.

[2] K. C. Kapur and L. R. Lamberson, Reliability in Engineering Design, John Wiley \& Sons, New York, NY, USA, 1977.

[3] R. K. Iyer and D. J. Rossetti, "Measurement-based model for workload dependence of CPU errors," IEEE Transactions on Computers, vol. C-35, no. 6, pp. 511-519, 1986.

[4] A. Barros, C. Bérenguer, and A. Grall, "Optimization of replacement times using imperfect monitoring information," IEEE Transactions on Reliability, vol. 52, no. 4, pp. 523-534, 2003.

[5] V. S. Amari, M. B. Krishna, and H. Pham, "Tampered failure rate load-sharing systems: status and perspectives," in Handbook of Performability Engineering, pp. 291-308, Springer, 2008.

[6] J. C. Fu, "Reliability of Consecutive-k-out-of-n:F Systems with (k-1)-step Markov Dependence," IEEE Transactions on Reliability, vol. 35, no. 5, pp. 602-606, 1986.

[7] G. Xiao and Z. Li, "Estimation of dependability measures and parameter sensitivities of a consecutive- $k$-out-of- $n$ : F repairable system with $(k-1)$-step Markov dependence by simulation," IEEE Transactions on Reliability, vol. 57, no. 1, pp. 71-83, 2008.

[8] W. Y. Yun, G. R. Kima, and H. Yamamoto, "Economic design of a circular consecutive-k-out-of-n: F system with (k-1)-step Markov dependence," IEEE Transactions on Reliability, vol. 57, pp. 464-478, 2008.

[9] S. Kotz, C. D. Lai, and M. Xie, "On the effect of redundancy for systems with dependent components," IIE Transactions (Institute of Industrial Engineers), vol. 35, no. 12, pp. 1103-1110, 2003.

[10] L. Y. Wang, X. J. Jia, and J. Zhang, "Reliability evaluation for multi-state markov repairable systems with redundant dependencies," Quality Technology and Quantitative Management, vol. 10, no. 3, pp. 277-289, 2013.

[11] M. Jain and R. Gupta, "Load sharing M-out of-N: G system with non-identical components subject to common cause failure," International Journal of Mathematics in Operational Research, vol. 4, no. 5, pp. 586-605, 2012.

[12] C.-Y. Li, X. Chen, X.-S. Yi, and J.-Y. Tao, "Heterogeneous redundancy optimization for multi-state series-parallel systems subject to common cause failures," Reliability Engineering and System Safety, vol. 95, no. 3, pp. 202-207, 2010.

[13] J. E. Ramirez-Marquez and D. W. Coit, "Optimization of system reliability in the presence of common cause failures," Reliability Engineering and System Safety, vol. 92, no. 10, pp. 1421-1434, 2007. 
[14] L. Xing, A. Shrestha, and Y. Dai, "Exact combinatorial reliability analysis of dynamic systems with sequence-dependent failures," Reliability Engineering and System Safety, vol. 96, no. 10, pp. 1375-1385, 2011.

[15] G. Levitin and L. Xing, "Reliability and performance of multistate systems with propagated failures having selective effect," Reliability Engineering and System Safety, vol. 95, no. 6, pp. 655661, 2010.

[16] G. Maaroufi, A. Chelbi, and N. Rezg, "Optimal selective renewal policy for systems subject to propagated failures with global effect and failure isolation phenomena," Reliability Engineering and System Safety, vol. 114, no. 1, pp. 61-70, 2013.

[17] L. Fiondella and L. Xing, "Discrete and continuous reliability models for systems with identically distributed correlated components," Reliability Engineering and System Safety, vol. 133, pp. $1-10,2015$.

[18] Y. Zhou, Z. Zhang, T. R. Lin, and L. Ma, "Maintenance optimisation of a multi-state series-parallel system considering economic dependence and state-dependent inspection intervals," Reliability Engineering and System Safety, vol. 111, pp. 248259, 2013.

[19] L. Wang and L. Cui, "Aggregated semi-Markov repairable systems with history-dependent up and down states," Mathematical and Computer Modelling, vol. 53, no. 5-6, pp. 883-895, 2011.

[20] L. Y. Wang and S. B. Si, "Reliability analysis of circular markov repairable systems with spatial dependence," Journal of Northwestern Polytechnical University, vol. 32, no. 6, pp. 923-928, 2014 (Chinese).

[21] L. Y. Wang, Y. R. Tian, Y. L. Wang, and B. Y. Liu, "A 6component circular multi-state Markov repairable system with spatial dependence," in Proceedings of the 1st International Conference on Reliability Systems Engineering (ICRSE '15), pp. 1-5, IEEE, Beijing, China, October 2015.

[22] F. Ball, R. K. Milne, and G. F. Yeo, "Multivariate semi-Markov analysis of burst properties of multiconductance single ion channels," Journal of Applied Probability, vol. 39, no. 1, pp. 179196, 2002.

[23] D. V. Widder, The Laplace Transform, Princeton Mathematical Series, Princeton University Press, Princeton, NJ, USA, 1946.

[24] A. Lisnianski and G. Levitin, Multi-State System Reliability: Assessment, Optimization and Applications, World Scientific, Singapore, 2003. 


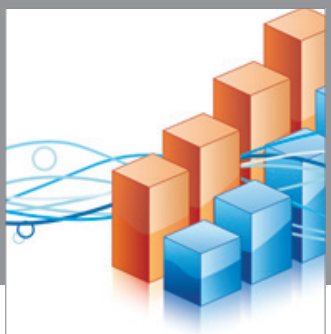

Advances in

Operations Research

vatem alat4

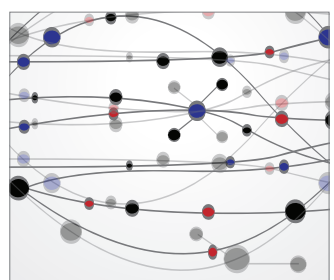

\section{The Scientific} World Journal
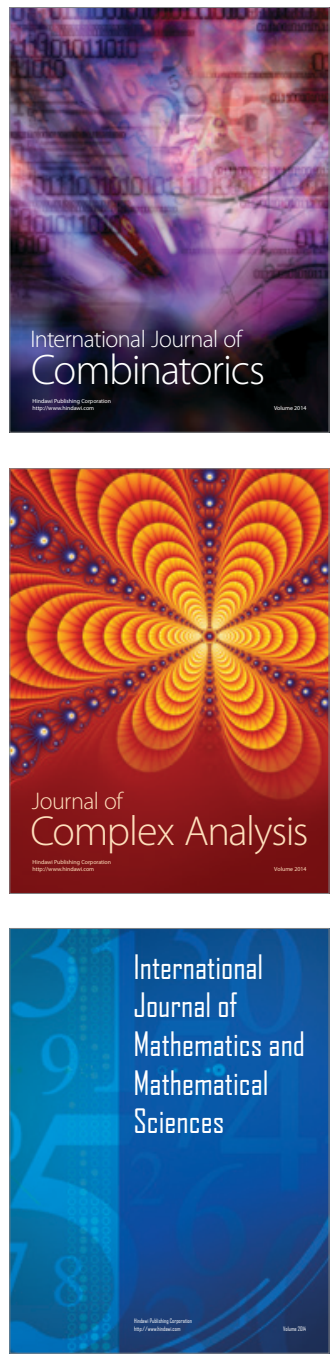
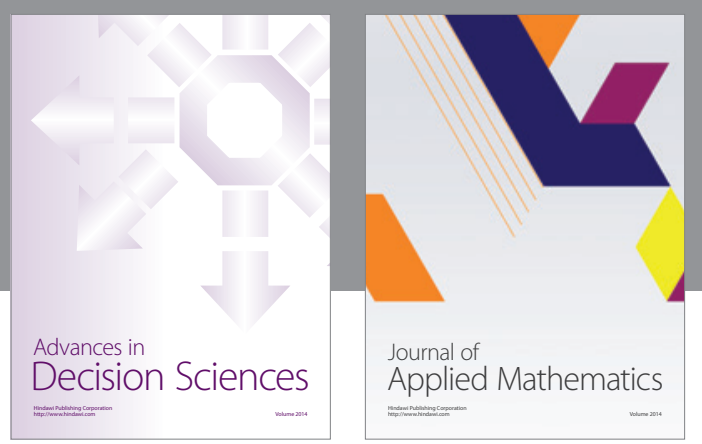

Algebra

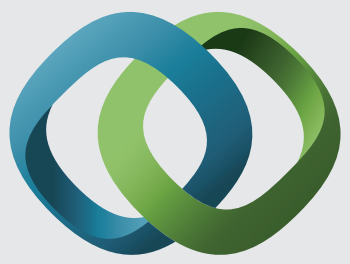

\section{Hindawi}

Submit your manuscripts at

https://www.hindawi.com
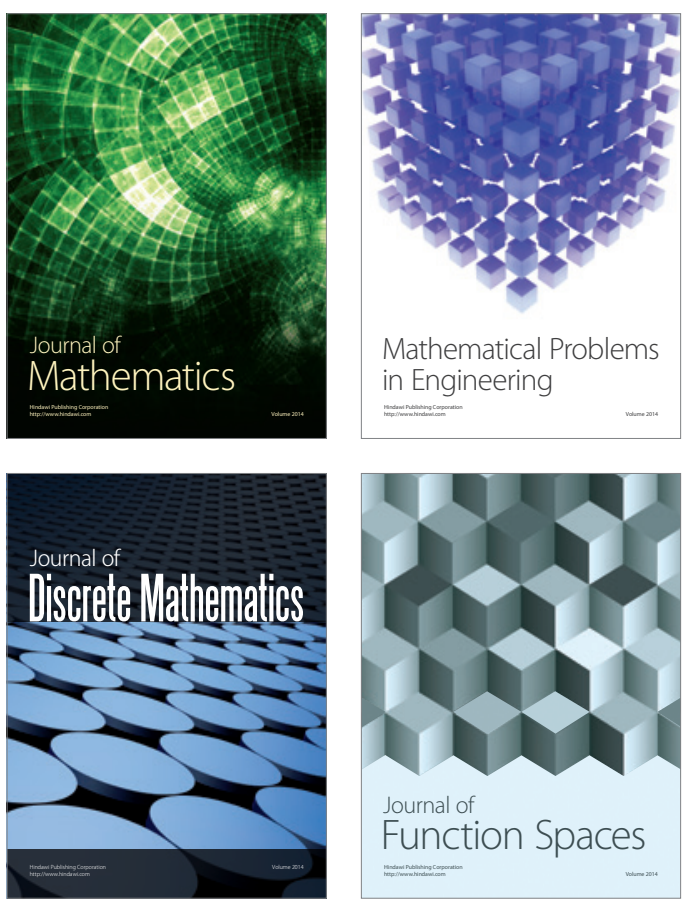

Mathematical Problems in Engineering
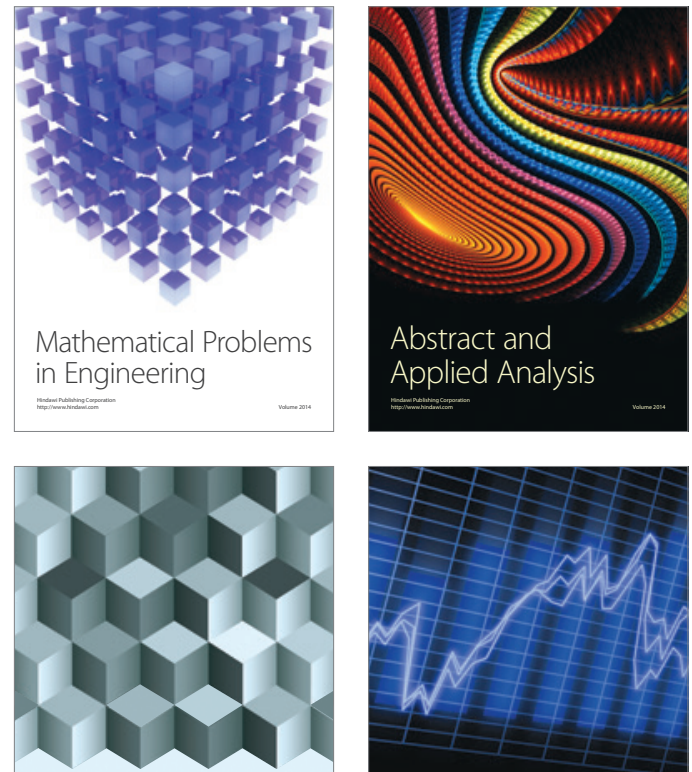

Journal of

Function Spaces

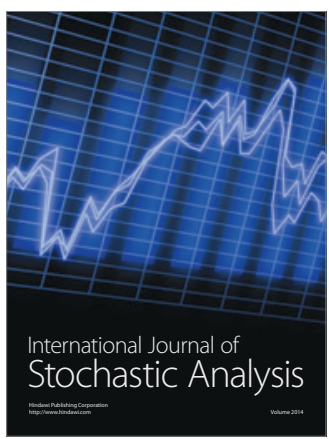

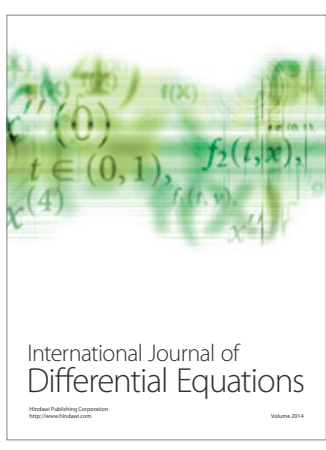
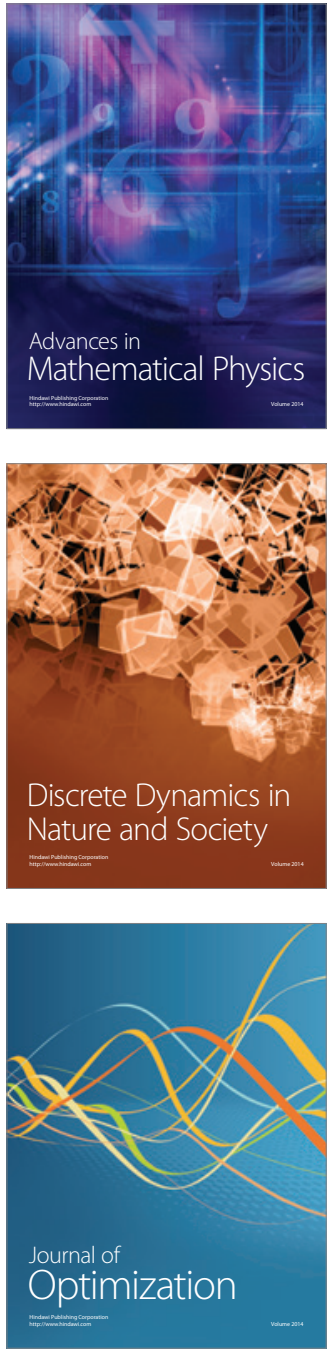